\title{
Evaluation of current prediction models for Lynch syndrome: updating the PREMM5 model to identify PMS2 mutation carriers
}

\author{
A. Goverde ${ }^{1,2}$ (1) M. C. W. Spaander ${ }^{2}$ D. Nieboer ${ }^{3}$ A. M. W. van den Ouweland ${ }^{1}$. \\ W. N. M. Dinjens ${ }^{4} \cdot$ H. J. Dubbink ${ }^{4} \cdot$ C. J. Tops ${ }^{5} \cdot$ S. W. ten Broeke ${ }^{5} \cdot$ M. J. Bruno ${ }^{2}$. \\ R. M. W. Hofstra ${ }^{1}$ E. W. Steyerberg ${ }^{6}$ A. Wagner ${ }^{1,7}$
}

Published online: 20 September 2017

(C) The Author(s) 2017. This article is an open access publication

\begin{abstract}
Until recently, no prediction models for Lynch syndrome (LS) had been validated for PMS2 mutation carriers. We aimed to evaluate MMRpredict and PREMM5 in a clinical cohort and for PMS2 mutation carriers specifically. In a retrospective, clinic-based cohort we calculated predictions for LS according to MMRpredict and PREMM5. The area under the operator receiving characteristic curve (AUC) was compared between MMRpredict and PREMM5 for LS patients in general and for different LS genes specifically. Of 734 index patients, $83(11 \%)$ were diagnosed with LS; 23 MLH1, 17 MSH2, 31 MSH6 and 12 PMS2 mutation carriers. Both prediction models performed well for $M L H 1$ and MSH2 (AUC 0.80 and 0.83 for PREMM5 and 0.79 for
\end{abstract}

Electronic supplementary material The online version of this article (doi:10.1007/s10689-017-0039-1) contains supplementary material, which is available to authorized users.

$\triangle$ A. Wagner

a.wagner@erasmusmc.nl

1 Department of Clinical Genetics, Erasmus MC, University Medical Center, Rotterdam, The Netherlands

2 Department of Gastroenterology and Hepatology, Erasmus MC, University Medical Center, Rotterdam, The Netherlands

3 Department of Public Health, Erasmus MC, University Medical Center, Rotterdam, The Netherlands

4 Department of Pathology, Erasmus MC, University Medical Center, Rotterdam, The Netherlands

5 Department of Clinical Genetics, Leiden University Medical Center, Leiden, The Netherlands

6 Department of Medical Statistics and Bioinformatics, Leiden University Medical Center, Leiden, The Netherlands

7 Department of Clinical Genetics, Erasmus MC, University Medical Center, Room Ee-2018, P. O. Box 2040, 3000 CA Rotterdam, The Netherlands
MMRpredict) and fair for MSH6 mutation carriers (0.69 for PREMM5 and 0.66 for MMRpredict). MMRpredict performed fair for PMS2 mutation carriers (AUC 0.72), while PREMM5 failed to discriminate $P M S 2$ mutation carriers from non-mutation carriers (AUC 0.51). The only statistically significant difference between PMS2 mutation carriers and non-mutation carriers was proximal location of colorectal cancer ( 77 vs. $28 \%, \mathrm{p}<0.001$ ). Adding location of colorectal cancer to PREMM5 considerably improved the models performance for PMS2 mutation carriers (AUC 0.77) and overall (AUC 0.81 vs. 0.72). We validated these results in an external cohort of 376 colorectal cancer patients, including 158 LS patients. MMRpredict and PREMM5 cannot adequately identify PMS2 mutation carriers. Adding location of colorectal cancer to PREMM5 may improve the performance of this model, which should be validated in larger cohorts.

Keywords Lynch syndrome - Prediction models . Colorectal cancer $\cdot$ Hereditary cancer

\section{Introduction}

Lynch syndrome (LS) is a hereditary predisposition to colorectal cancer, endometrial cancer and other extra-colonic cancers at a young age $[1,2]$. Morbidity and mortality of LS carriers can be significantly reduced by surveillance programs [3-5]. Therefore identifying LS carriers is of great importance.

LS is caused by a germline mutation in one of the mismatch repair (MMR) genes $M L H 1, M S H 2, M S H 6$ or $P M S 2$, or in the $3^{\prime}$ end of the EPCAM gene and consequent hypermethylation of the $M S H 2$ promoter region [6-10]. As a result, tumours in LS patients are characterized by 
microsatellite instability (MSI) and by loss of MMR protein expression in immunohistochemistry (IHC) [11-13]. Analysis of MSI and IHC, combined with $M L H 1$ promoter methylation analysis to exclude sporadic MMR deficient tumours, are used to identify patients with tumours likely caused by LS [13]. A definite diagnosis of LS is made when a pathogenic germline mutation is found.

The revised Bethesda guidelines were based on a set of diagnostic criteria to select patients eligible for LS screening in tumour tissue. However, due to limited sensitivity, many LS patients will likely be missed by these guidelines [14-17]. Several prediction models, such as MMRpro, MMRpredict and PREMM5 have also been developed to calculate an individual's probability of carrying a germline MMR mutation [18-20]. These models could aid in the selection of patients at high risk of having LS, for tumour analysis or direct germline mutation analysis. MMRpro is less useful in clinical practice since detailed information of all relatives is needed as input for the model [19]. However, MMRpredict and PREMM P $_{1,2,6}$ (a previous version of the newly developed PREMM $_{5}$ model) both performed well in previous evaluations [21-27]. An advantage of PREMM5 is that it can also be used for individuals with extracolonic malignancies and healthy individuals, as opposed to MMRpredict, which can only be used for CRC patients. Until recently, all prediction models for LS were developed with cohorts of patients carrying a $M L H 1, M S H 2$, or $M S H 6$ mutation. The recently published PREMM5 model is the only model that included PMS2 mutation carriers in its development.

In this study we aimed to evaluate MMRpredict and PREMM5 in a clinical cohort and for PMS2 mutation carriers specifically. Additionally, we aimed to identify clinical features useful for distinguishing PMS2 mutation carriers from non-mutation carriers.

\section{Methods}

In a retrospective, clinic-based cohort we assessed the performance of MMRpredict and PREMM5 in predicting LS mutations in general and for MLH1, MSH2, MSH6 and PMS2 mutations specifically. Additionally, we performed a univariate analysis to identify variables that can distinguish PMS2 mutation carriers from patients with no MMR mutation.

\section{Study population}

We collected data for all families that were referred for genetic counselling at Erasmus MC, Rotterdam, The Netherlands, and in which colorectal cancer was analysed for MSI and/or IHC between 2000 and 2010. Exclusion criteria were: failed or inconclusive analysis for MSI and IHC, a pathogenic mutation in APC or MUTYH, a variant of unknown clinical significance in one of the MMR genes or $A P C$, and MSI or IHC suspect for LS while no MMR mutation was detected. To increase the number of LS families, 35 LS families outside our cohort, diagnosed after 2010, were also included in the analysis.

\section{Analysis of MSI and IHC}

MSI analysis was carried out with five markers for MSI as described previously; up to 2007 the Bethesda panel [28] was used and from 2007 onwards our center performs Promega pentaplex MSI analysis [29]. IHC for MLH1, MSH2, MSH6 and PMS2 protein was performed as described previously [13]. Tumours without MSI or only a low degree of MSI and with all MMR proteins present, were considered MMR proficient. Tumours showing a high degree of MSI and/or absence of one or more MMR proteins, were considered MMR deficient. MLH1 hypermethylation analysis was performed to distinguish between sporadic MMR deficient tumours and MMR deficient tumours suspect for LS.

\section{Germline mutation analysis}

Patients with MMR deficient tumours suspect for LS underwent germline mutation analysis of the gene indicated by IHC. Germline mutation analysis of $M L H 1, M S H 2$ and MSH6 was performed by sequencing and multiplex ligation dependent probe amplification analyses. PMS2 mutation analysis was performed as described elsewhere [30].

\section{Family classification}

Tumour characteristics, age at diagnosis, results of molecular diagnostics and germline mutation analysis, and a detailed family history were collected from medical records. In every family the patient in whom MSI and/or IHC was analysed, was labelled the index patient. If more than one family member was screened for LS, the youngest CRC patient analysed was considered the index patient. Index patients with MMR proficient tumours or sporadic MMR deficient tumours, were labelled non-mutation carriers. Families identified with a pathogenic MMR mutation were labelled LS families.

\section{Prediction models}

For each index patient the probability of carrying a LS mutation according to MMRpredict and PREMM5 was calculated as previously described [18, 20].

For PREMM5, the equation was slightly different from the published equation, based on personal communications 
with F. Kastrinos. See Supplemental Material (Appendix 1) for the corrected PREMM5 equation.

\section{Statistical analysis}

Data were analyzed using SPSS statistical software version 21.0. Differences between mutation carriers and non-mutation carriers were compared using the Chi square test or Fishers' exact test for frequencies, and by using the Mann Whitney $U$ test for continuous data. These analysis were also performed to compare PMS2 mutation carriers with non-mutation carriers. $\mathrm{P}$ values $<0.01$ were considered statistically significant.

Receiver operating characteristic curves were created for MMRpredict and PREMM5 by plotting the true positive rate (sensitivity) against the false positive rate (1specificity). Performance of MMRpredict and PREMM5 was evaluated by the area under the receiver operating characteristic curve (AUC). We compared the AUC of PREMM5 and MMRpredict for LS patients in general and for the different MMR genes specifically. Sensitivity and specificity were calculated for cut-offs previously indicated by the developers of the models $(5,10,20$ and $40 \%$ ). These values were compared with the sensitivity and specificity of the revised Bethesda guidelines.

\section{Model updating}

Location of CRC is included in MMRpredict, but not in the PREMM5 model. To update the PREMM5 model, we used a previously proposed framework to update multinomial logistic regression models [31]. We extended the PREMM5 model using recalibration and extension. The PREMM5 model contains four linear predictors, each contributing weights to the probability of carrying a mutation in MLH1, MSH2 (or TACSTD1), MSH6 and PMS2. The coefficients of the linear predictors were constrained such that the linear predictor only contributed to the calculation of the corresponding mutation. Since the original PREMM5 model was developed on a population with no MSH6 mutation carriers with two or more CRCs, we developed two adaptations of the PREMM5 model. First we recalibrated the PREMM5 model and re-estimated the coefficient of the predictor 'Two or more CRCs' in the linear predictor for MSH6. In the second adaptation we also added side of CRC as an additional predictor to the original PREMM5 model. Discriminative ability of the prediction models was quantified using the AUC. Calculations were done using $\mathrm{R}$ software (version 3.3.0), with estimation of the coefficients in the updated PREMM5 model using the VGAM package.

\section{Validation of the extended PREMM5 model}

For external validation of the extended PREMM5 model, we used a cohort of $376 \mathrm{CRC}$ patients. Of these patients, 218 were patients with MMR proficient CRC, that where analysed in the Erasmus Medical Center Rotterdam outside the dates of our initial cohort. LS patients $(n=158)$ in our validation cohort were CRC patients from Leiden University Medical Center in whom an MMR mutation was found and with known location of CRC. For all patients of the validation cohort we calculated the probability of carrying an MMR mutation according to the original PREMM5 model and the extended model. The performance of both models were evaluated by comparing the AUC.

\section{Results}

A total of 734 index patients were included in the study; $346(47 \%)$ were male and mean age at time of diagnosis was 53 years ( \pm 13 years). Overall, $569(78 \%)$ patients fulfilled the revised Bethesda guidelines. Of the 734 index patients, 83 (11\%) were diagnosed with a LS mutation; $23 \mathrm{MLH1}, 17$ MSH2, 31 MSH6 and 12 PMS2 mutation carriers.

\section{Patient characteristics}

Patient characteristics for mutation-positive and mutationnegative patients are shown in Table 1 . Significantly more mutation carriers developed multiple CRCs ( 21 vs. $10 \%$, $\mathrm{p}=0.005)$ and multiple LS-associated cancers in general (13 vs. $4 \%, \mathrm{p}=0.002)$ than non-mutation-carriers. CRC patients carrying an MMR mutation had a younger age of onset (49 vs. 53 years, $\mathrm{p}=0.002)$ and more often had proximal CRCs (64 vs. $28 \%, p<0.001$ ) than non-mutation carriers. Among women, the frequency of EC was higher for mutation carriers than for non-mutation carriers ( 41 vs. $3 \%$, p < 0.001$)$. In the mutation positive group, first and second degree relatives developed CRC at a younger age than in the mutation negative group (50 vs. 64 years, $\mathrm{p}<0.001$ and 47 vs. 62 years, $\mathrm{p}=0.008$ ). First degree relatives of mutation carriers had higher rates of EC than relatives of non-mutation carriers (19 vs. $5 \%, \mathrm{p}<0.001)$.

\section{Discriminative ability of prediction models}

Overall, PREMM5 predicted higher probabilities of carrying a LS mutation than MMRpredict (median score 0.06 vs. 0.03 , Supplemental Table 1). For mutation carriers, risk scores varied from 0.02 to 0.99 for PREMM5 and from 0.002 to 0.99 for MMRpredict. Both prediction models could fairly discriminate between index patients with and without an MMR mutation.(Fig. 1) PREMM5 
Table 1 Index characteristics and family history by mutation status $(\mathrm{n}=734)$

\begin{tabular}{|c|c|c|c|}
\hline & Mutation negative, $\%$ (n) & Mutation positive, $\%$ (n) & $\mathrm{P}$ value \\
\hline $\mathrm{n}$ & 651 & 83 & \\
\hline Revised Bethesda guidelines & $76 \%$ (494) & $90 \%(75)$ & 0.003 \\
\hline \multicolumn{4}{|l|}{ Index characteristics } \\
\hline Male gender & $47 \%(305)$ & $49 \%(41)$ & 0.66 \\
\hline \multicolumn{4}{|l|}{$\mathrm{CRC}$} \\
\hline Age CRC (median, IQR) & 53 years $[45-62]$ & 49 years [39-59] & 0.002 \\
\hline Proximal CRC & $28 \%(185)$ & $64 \%(53)$ & $<0.001$ \\
\hline$\geq 2 \mathrm{CRCs}$ & $10 \%(66)$ & $21 \%(17)$ & 0.005 \\
\hline Endometrial cancer & $3 \%(11)$ & $41 \%(17)$ & $<0.001$ \\
\hline Age EC (median, IQR) & 55 years $[50-75]$ & 54 years [49-57] & 0.18 \\
\hline Multiple LS cancers & $4 \%(27)$ & $13 \%(11)$ & 0.002 \\
\hline \multicolumn{4}{|l|}{ First degree relatives } \\
\hline $\mathrm{CRC}$ & $55 \%(358)$ & $51 \%(42)$ & 0.45 \\
\hline$\geq 2$ FDRs with CRC & $16 \%(107)$ & $17 \%(14)$ & 0.92 \\
\hline Age CRC (median, IQR) & 64 years $[55-71]$ & 50 years [43-57] & $<0.001$ \\
\hline Endometrial cancer & $5 \%(35)$ & $19 \%(16)$ & $<0.001$ \\
\hline$\geq 2$ FDRs with EC & $0.6 \%(4)$ & $2 \%(2)$ & 0.14 \\
\hline Age EC (median, IQR) & 55 years $[50-64]$ & 50 years [45-57] & 0.25 \\
\hline Other LS cancers & $22 \%(142)$ & $19 \%(16)$ & 0.60 \\
\hline \multicolumn{4}{|l|}{ Second degree relatives } \\
\hline $\mathrm{CRC}$ & $33 \%(212)$ & $35 \%(29)$ & 0.66 \\
\hline$\geq 2$ SDRs with CRC & $12 \%(81)$ & $12 \%(10)$ & 0.92 \\
\hline Age CRC (median, IQR) & 62 years $[50-74]$ & 47 years [38-64] & 0.008 \\
\hline Endometrial cancer & $3 \%(22)$ & $7 \%(6)$ & 0.12 \\
\hline$\geq 2$ SDRs with EC & $0.3 \%(2)$ & $2 \%(2)$ & 0.07 \\
\hline Age EC (median, IQR) & 70 years $[50-76]$ & 49 years [44-51] & 0.13 \\
\hline Other LS cancers & $16 \%(104)$ & $18 \%(15)$ & 0.63 \\
\hline
\end{tabular}

and MMRpredict had similar overall performance (AUC 0.72 [95\% CI 0.66-0.79] vs. 0.73 [95\% CI 0.66-0.79]). For MLH1 and MSH2 mutation carriers, both prediction models performed well, with AUC of 0.80 [95\% CI 0.71-0.89] and 0.83 [95\% CI 0.73-0.94] for PREMM5 and AUC of 0.79 [95\% CI 0.69-0.89 and 0.67-0.91] for MMRpredict. Both models had a fair discriminative power for MSH6 mutation carriers (AUC of 0.69 [95\% CI 0.58-0.80] for PREMM5 and AUC of 0.66 [95\% CI 0.56-0.76] for MMRpredict). MMRpredict still had fair performance for PMS2 mutation carriers (AUC of 0.72 [95\% CI 0.57-0.87]), while PREMM5 failed to discriminate PMS2 mutation carriers from non-mutation carriers at all with an AUC of 0.51 [95\% CI 0.35-0.66].

\section{Sensitivity and specificity}

Using a cut-off of 5\% for both prediction models, PREMM5 had a higher sensitivity than MMRpredict (78 vs. 70\%). This higher sensitivity came at the expense of a lower specificity (46 vs. 67\%). For PREMM5, using a cut-off of 5\%, resulted in a sensitivity for $M L H 1$ and $M S H 2$ mutations of 88 and $91 \%$, while the sensitivity for MSH6 mutation carriers was
74\% and the sensitivity for $P M S 2$ mutation carriers was only $50 \%$. For MMRpredict, at a 5\% cut-off sensitivity for $\mathrm{MLH1}$ and $\mathrm{MSH} 2$ mutation carriers were 74 and $77 \%$, while sensitivity for PMS2 as well as MSH6 mutation carriers were 65 and $67 \%$. For both models, using a cut-off of $\geq 20 \%$ failed to identify over $50 \%$ of the mutation carriers.

Sensitivity of the revised Bethesda guidelines decreased from $96 \%$ for $M L H 1$ mutation carriers to $83 \%$ for $P M S 2$ mutation carriers (Supplemental Table 2). Overall, the revised Bethesda guidelines had a sensitivity of $90 \%$ with a specificity of $24 \%$. In order to reach the same sensitivity, PREMM5 and MMRpredict had a similar specificity (25\%).

\section{PMS2 mutation carriers versus non-mutation carriers}

Mutation carriers differed significantly from non-mutation carriers in many ways (Table 1 ). In contrast, there were almost no significant differences between PMS2 mutation carriers and non-mutation carriers. Only one significant difference remained; PMS2 mutation carriers more often had proximal CRC than patients without an MMR mutation (83 vs. $28 \%$, p $<0.001$ ) (Table 2). 

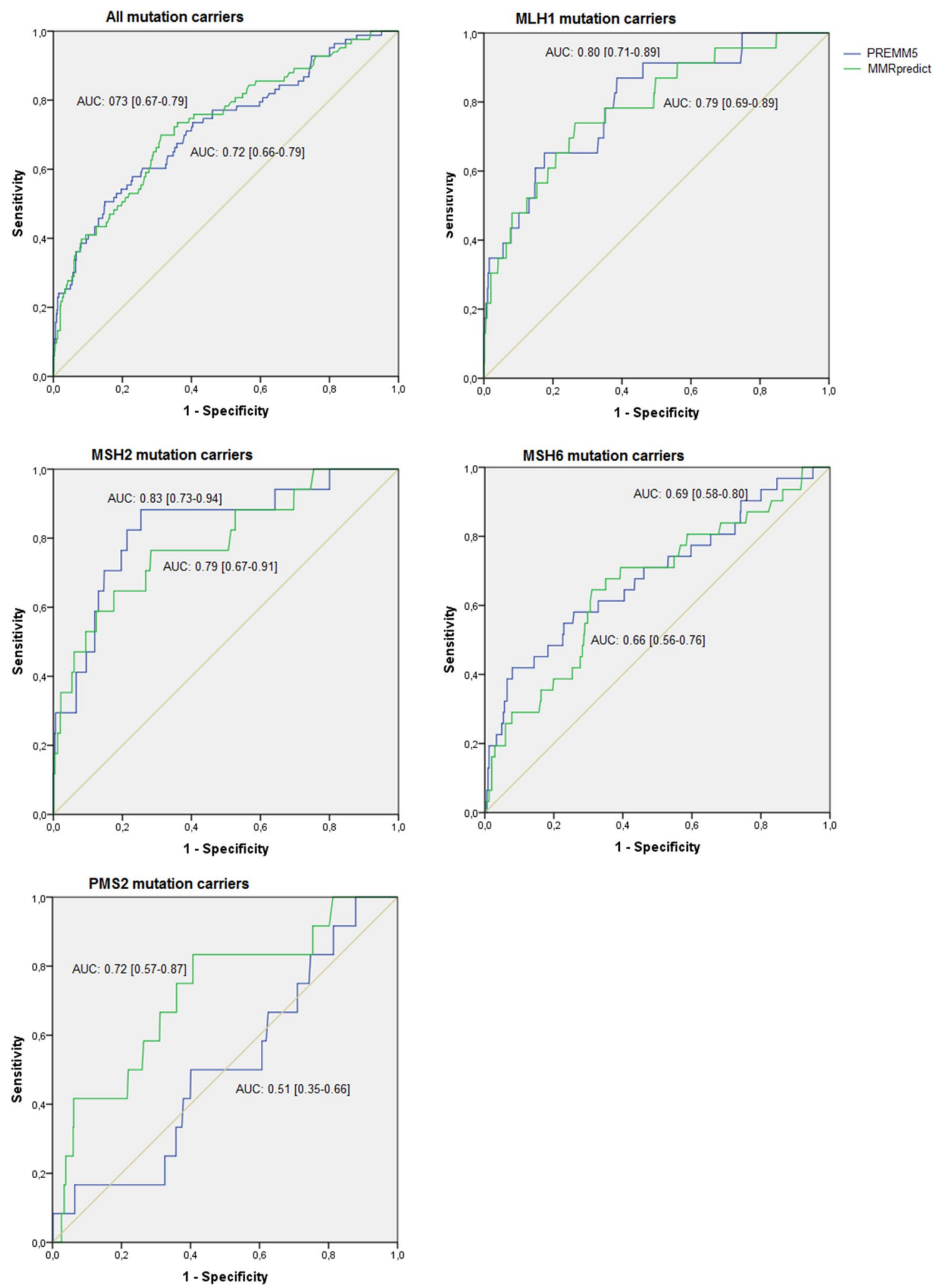

Fig. 1 Performance of PREMM5 and MMRpredict in a clinical setting for all mutation carriers and for individual MMR mutations 
Table 2 Index characteristics and family history for PMS2 mutation carriers compared with non-mutation carriers

\begin{tabular}{|c|c|c|c|}
\hline & Mutation negative, $\%$ (n) & $\begin{array}{l}\text { PMS2 mutation posi- } \\
\text { tive, } \%(\mathrm{n})\end{array}$ & $\mathrm{P}$ value \\
\hline $\mathrm{n}$ & 651 & 12 & \\
\hline Revised Bethesda guidelines & $76 \%(494)$ & $83 \%(10)$ & 0.74 \\
\hline \multicolumn{4}{|l|}{ Index characteristics } \\
\hline Male gender & $47 \%(305)$ & $50 \%(6)$ & 0.83 \\
\hline \multicolumn{4}{|l|}{$\mathrm{CRC}$} \\
\hline Age CRC (median, IQR) & 53 years [45-62] & 46 years [40-61] & 0.21 \\
\hline Proximal CRC & $28 \%(185)$ & $83 \%(10)$ & $<0.001$ \\
\hline$\geq 2 \mathrm{CRCs}$ & $10 \%(66)$ & $8 \%(1)$ & 1.0 \\
\hline Endometrial cancer & $3 \%(11)$ & $0 \%(0)$ & 1.0 \\
\hline Age EC (median, IQR) & 55 years $[50-75]$ & & \\
\hline Multiple LS cancers & $4 \%(27)$ & $0 \%(0)$ & 1.0 \\
\hline \multicolumn{4}{|l|}{ First degree relatives } \\
\hline CRC & $55 \%(358)$ & $42 \%(5)$ & 0.36 \\
\hline$\geq 2$ FDRs with CRC & $16 \%(107)$ & $8 \%(1)$ & 0.70 \\
\hline Age CRC (median, IQR) & 64 years $[55-71]$ & 62 years [45-90] & 0.68 \\
\hline Endometrial cancer & $5 \%(35)$ & $17 \%(2)$ & 0.14 \\
\hline$\geq 2$ FDRs with EC & $0.6 \%(4)$ & $8 \%(1)$ & 0.88 \\
\hline Age EC (median, IQR) & 55 years $[50-64]$ & 37 years $[-]$ & 0.24 \\
\hline Other LS cancers & $22 \%(142)$ & $8 \%(1)$ & 0.48 \\
\hline \multicolumn{4}{|l|}{ Second degree relatives } \\
\hline CRC & $33 \%(212)$ & $17 \%(2)$ & 0.35 \\
\hline$\geq 2$ SDRs with CRC & $12 \%(81)$ & $8 \%(1)$ & 1.0 \\
\hline Age CRC (median, IQR) & 62 years $[50-74]$ & 39 years [39-] & 0.12 \\
\hline Endometrial cancer & $3 \%(22)$ & $8 \%(1)$ & 0.35 \\
\hline$\geq 2$ SDRs with EC & $0.3 \%(2)$ & $8 \%(1)$ & 0.05 \\
\hline Age EC (median, IQR) & 70 years $[50-76]$ & 49 years $[-]$ & 0.67 \\
\hline Other LS cancers & $16 \%(104)$ & $17 \%(2)$ & 1.0 \\
\hline
\end{tabular}

\section{Improvement of the PREMM5 model}

Since location of CRC was the only significant difference between PMS2 mutation carriers and non-mutation carriers, we incorporated this variable in the PREMM5 model, aiming to improve the prediction model. For PMS2 mutation carriers, the extended PREMM5 model had considerably better predictions than the original PREMM $5_{5}$ model (AUC 0.77 [95\% CI 0.63-0.90] vs. 0.51 [95\% CI 0.35-0.66]) (Fig. 2). At a 5\% cut-off, the new PREMM5 model identified $5 / 6$ PMS2 mutation carriers that would have been missed by PREMM5 and 3/4 PMS2 mutation carriers that would have been missed by MMRpredict at the same cut-off.

Adding tumour location also improved the performance of PREMM5 for identifying $M L H 1$ (AUC 0.92 [95\% CI $0.88-0.97$ ] vs. 0.80 [95\% CI 0.71-0.89]) and MSH6 (AUC 0.75 [95\% CI $0.65-0.84]$ vs. 0.69 [95\% CI $0.58-0.80]$ ]) mutation carriers (Fig. 2). However, performance for MSH2 mutation carriers slightly decreased (AUC 0.80 [95\% CI 0.69-0.91] vs. 0.83 [95\% CI 0.73-0.94]). Overall, the adjusted PREMM5 model performed better than the original
PREMM5 model (AUC 0.81 [95\% CI 0.76-0.86] vs. 0.72 [95\% CI 0.66-0.79]) and MMRpredict (AUC 0.81 vs. 0.73 [95\% CI 0.66-0.79]). The adjusted prediction model can be found as supplemental material.

At a 5\% cut-off, sensitivity of the extended PREMM5 model was higher than the sensitivity of the original PREMM5 model (92 vs. 78\%) with similar specificity (45 vs. $46 \%$ ). Sensitivity and specificity of the extended PREMM5 model at a 5\% cut off were both higher than those of the revised Bethesda guidelines (sensitivity 92 vs. $90 \%$ and specificity 45 vs. $24 \%$ ).

\section{Validation of the extended PREMM5 model}

In our validation cohort, $60 \%$ of the patients were male and median age was 55 years (IQR 45-63 years). The cohort included $31 \mathrm{MLH1}, 26 \mathrm{MSH} 2,28 \mathrm{MSH} 6$ and 73 PMS2 mutation carriers. Similar to the results in the initial cohort, the extended PREMM5 model had better predictions than the original PREMM5 model for PMS2 mutation carriers (AUC 0.90 [95\% CI 0.86-0.94] vs. 0.82 [95\% CI 0.76-0.87]) and 

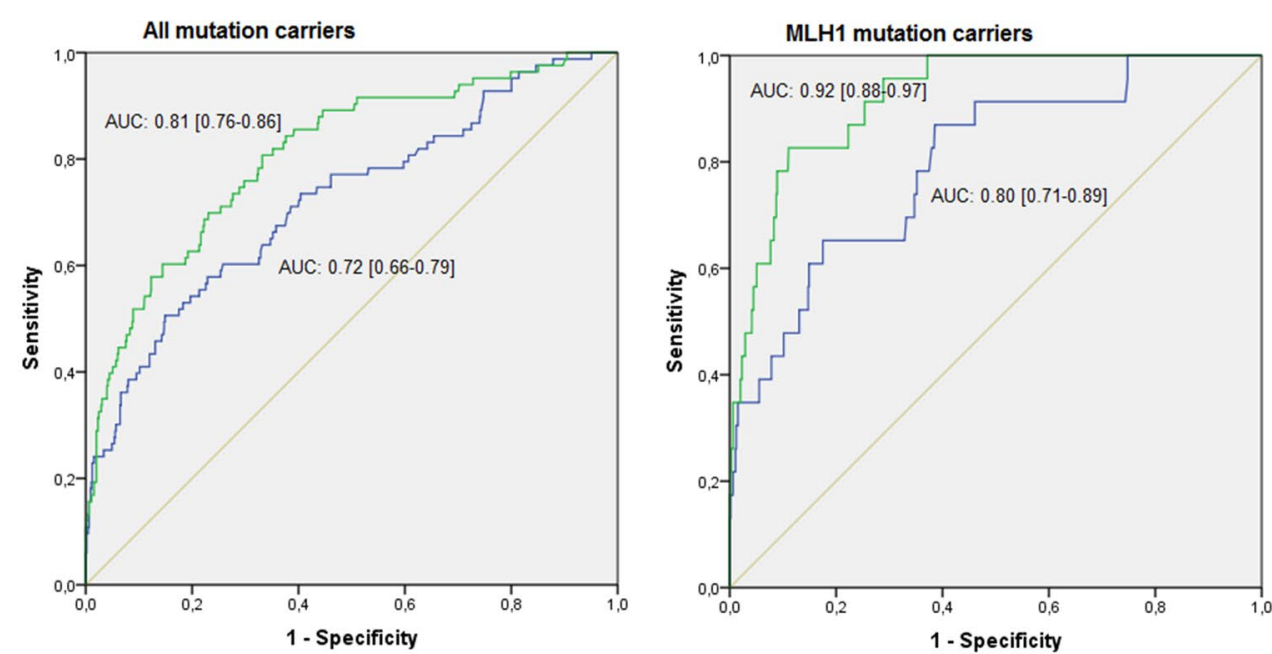

- PREMM5

- extended PREMM5
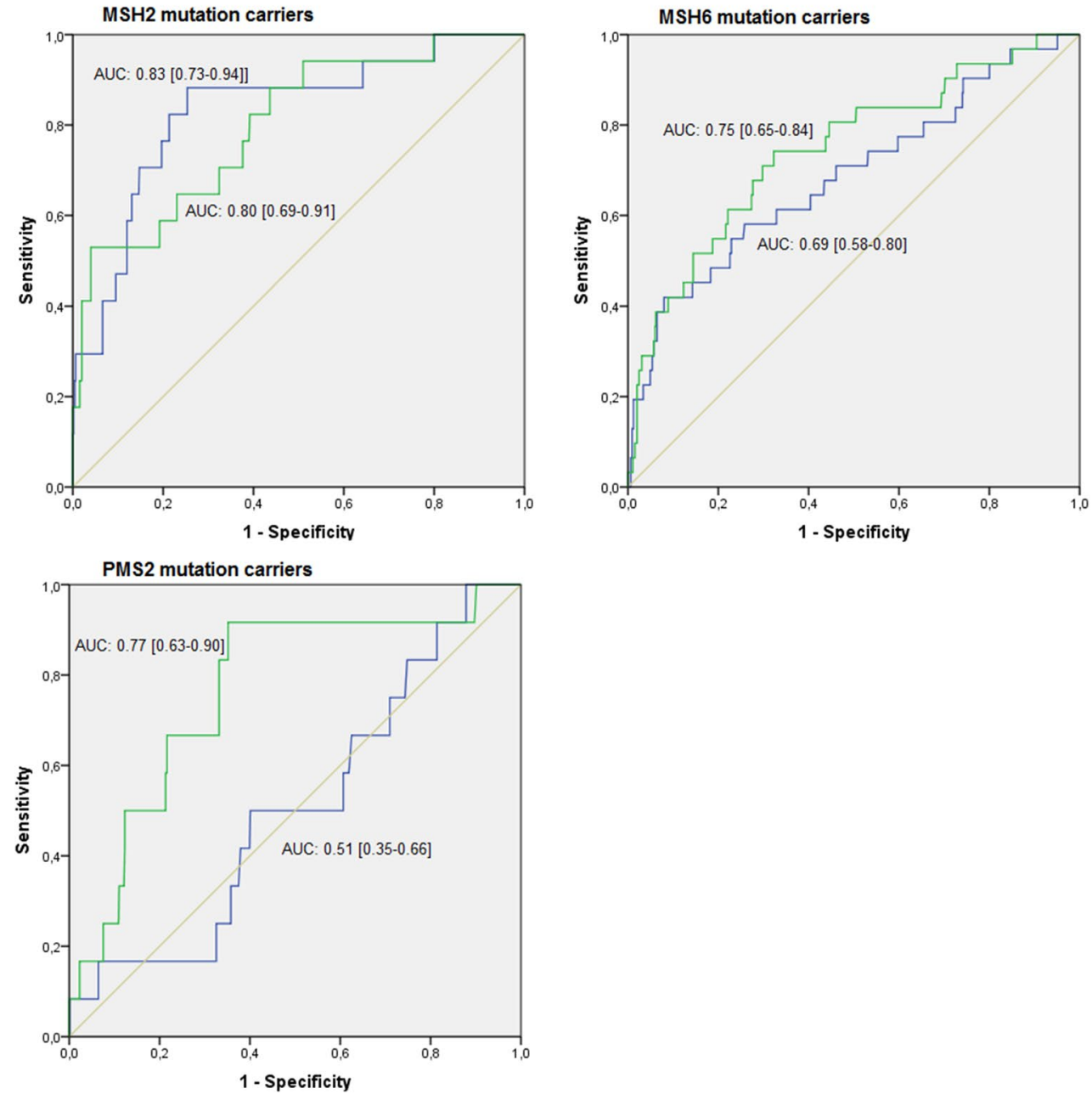

Fig. 2 Performance of PREMM5 and the extended PREMM5 model in a clinical setting for all mutation carriers and for individual MMR mutations

overall (AUC 0.92 [95\% CI 0.89-0.95] vs. 0.87 [95\% CI 0.84-0.91]). Performance for MLH1, MSH2 and MSH6 mutation carriers was also slightly better for the extended
PREMM5 model than for the original PREMM5 model (AUC 0.97 [95\% CI 0.94-1.00] vs. 0.95 [95\% CI 0.91-0.99] for $M L H 1,0.97$ [95\% CI $0.93-1.00]$ vs. 0.96 [95\% CI 
$0.92-0.99$ ] for $M S H 2$ and 0.86 [95\% CI $0.97-0.93$ ] vs. 0.85

[95\% CI 0.77-0.93] for MSH6 mutation carriers).

\section{Discussion}

The results of our study indicate that while the models MMRpredict and PREMM5 can adequately predict whether an individual is likely to have Lynch syndrome, they fail to identify PMS2 mutation carriers. The performance of the PREMM5 model improved considerably by adding the location of CRC to the model. In our clinical cohort of 734 CRC patients as well as in a validation cohort of 376 CRC patients, this extended PREMM5 model not only identified $P M S 2$ mutation carriers more accurately, its overall performance was also better than the original PREMM5 model and the MMRpredict model.

Our results are in line with those of previous studies, where the PREMM ${ }_{1,2,6}$ model had a slightly better overall performance than MMRpredict [22, 32, 33]. The first PREMM model, PREMM $_{1,2}$ also performed better than MMRpredict in several studies [23, 24], but had similar $[25,26]$ or less accurate [21] predictions in other studies. A recent meta-analysis also found pooled AUCs to be higher for the PREMM model than for MMRpredict (AUC 0.84 vs. 0.81) [27].

Although PREMM5 had better overall predictions, MMRpredict had a better performance for PMS2 mutation carriers specifically. An explanation for this could be that the location of CRC is incorporated in the MMRpredict model but not in the PREMM $_{5}$ model. Proximal location of CRC is a known predictor for Lynch syndrome and in our cohort was the only significant difference between PMS2 mutation carriers and non-mutation carriers. After adding this new variable to the existing PREMM5 ${ }_{5}$ model, this new model performed better than MMRpredict for PMS2 mutation carriers. The extended PREMM5 5 model also performed better than the original model for MLH1, MSH2 and MSH6 mutation carriers and had a better overall performance.

In our validation cohort, all AUCs were much higher than in our original cohort, including those for PMS2 mutation carriers. Selection of patients for analysis of MSI and IHC may have been less stringent at the Erasmus Medical Center Rotterdam than at the Leiden University Medical Center. Therefore, mutation carriers in our validation cohort, who were all from Leiden University Medical Center, may have had a family history more suspect for Lynch syndrome than family history of the patients in our original cohort. This could explain the higher AUCs in the validation cohort. However, in both cohorts we showed that the extended PREMM5 had better performance.

Prediction models for Lynch syndrome are not yet regularly used in current clinical practice. However, the US
Multi-Society Task Force on Colorectal Cancer recommends genetic evaluation if an individual's risk of carrying an MMR gene mutation is $\geq 5 \%$ according to one of the prediction models MMRpro, MMRpredict or PREMM [34]. The American guideline recommends that all CRC patients undergo routine screening for LS by analysis of MSI and IHC [34], while current European guidelines recommend such routine screening in at least all CRC patients up to 70 years of age [35]. A recent study demonstrated that routine screening for LS without an age cut-off is not cost-effective [36]. A strategy using prediction models might lower the cost of screening for LS. In fact, two cost-effectiveness analyses found that strategies including prediction models were more cost-effective than those involving direct tumour testing of all CRC patients, if these prediction models were perfectly implemented [36, 37]. Additionally, prediction models could also be used in cases where no tumour tissue is available or where tumour tissue analysis failed, to assess whether an individual should be analysed for a germline MMR mutation.

The US Multi-Society Task Force on Colorectal Cancer recommends the use of either PREMM, MMRpredict or MMRpro to assess the probability of an individual carrying an MMR mutation [34]. Since we did not include the MMRpro model in our analysis, we do not know how MMRpro would have performed in our cohort. However, MMRpro is less useful in clinical practice since extensive family data is needed as input for the model. Collection of this kind of data is very time consuming and therefore not suitable in clinical practice. PREMM5 and MMRpredict are web-based models that are easily accessible and therefore much easier to use. Also, multiple studies-including the recent metaanalysis-have shown MMRpro to have similar accuracy to PREMM $_{1,2,6}$ [21-27, 32].

Both PREMM5 and MMRpredict were far more accurate for $M L H 1$ and $M S H 2$ mutation carriers than for LS patients carrying a mutation in $M S H 6$ or PMS2. This finding is in line with a previous study that showed that carriers of mutations in MSH6 or PMS2 had lower risk scores than carriers of a mutation in $M L H 1$ or $M S H 2$ [21]. In our study, discrimination between non-mutation carriers and PMS2 mutation carriers was the least accurate, in line with its more limited penetrance.

Around 15\% of all Lynch syndrome cases are estimated to be caused by PMS2 mutations [38]. In our cohort, $14 \%$ (12/83) of the Lynch syndrome patients were PMS2 mutation carriers. To our knowledge, our study is the first to validate LS prediction models for PMS2 mutation carriers specifically since the development of the PREMM5 model. At a 5\% cut-off, our extended PREMM5 model was able to detect 5/6 PMS2 mutation carriers who would have been missed by the original PREMM5 model at the same cut-off. Identification of Lynch syndrome carriers is highly important, since 
this allows not only them, but also their family members carrying the same mutation, to undergo intensive surveillance in order to prevent the development of cancer. Our new model would also identify more Lynch syndrome patients overall than the original PREMM5 model.

The performance of prediction models can differ between high-risk settings and population-based cohorts. Further validation studies should indicate whether our results can be generalized to settings with patients at low to median risk of having Lynch syndrome. Since patients in our study cohort were all referred for genetic counselling, family histories were obtained in detail and in many cases also verified by medical documents. In other settings where patients are at lower risk of having Lynch syndrome, family history is not verified and might be less reliable. Therefore, prediction models should also be validated in population-based cohorts. However, in a meta-analysis, prediction models performed better in population-based cohorts than in clinicbased cohorts [27].

It is not known whether the current prediction models for Lynch syndrome are useful in non-Western populations. In a recent study among Korean patients, PREMM $_{1,2,6}$ was more accurate than MMRpro and MMRpredict, but still only reached an AUC of 0.71 [32]. There was no association between tumour location and mutation status, so our extended PREMM5 model might not improve predictions in populations of non-Western ethnicity. However, germline analysis for $P M S 2$ was not performed in the Korean study, so there might have been more mutation carriers in their cohort. Another non-Western population has been studied by Khan et al., who analysed the performance of prediction models in 15 African American patients [22]. In these patients, MMRpredict and PREMM ${ }_{1,2,6}$ both had a high AUC of 0.89.

A main strength of our study was the large cohort, which consisted of more than 700 index patient including 83 Lynch syndrome patients. Also, our cohort included patients with MSH6 and PMS2 mutations. Since 12 patients were identified as a PMS2 mutation carrier, we were able to evaluate the prediction models for each MMR mutation specifically, admittedly with considerable uncertainty [39]. Furthermore, we validated the extended PREMM5 model in a separate cohort of 376 patients including 73 PMS2 mutation carriers.

A limitation of our study was that germline mutation analysis was not done for all index patients. Patients who had microsatellite stable tumours with normal IHC were assumed to be non-mutation carriers. However, some of these patients might still have an MMR mutation. Also, the sample size per gene was still relatively small and it is unclear whether our results from a high-risk population apply to a population-based setting.

In conclusion, we have shown that although MMRpredict and PREMM5 can accurately predict an individual's risk of carrying a causative MMR mutation, neither model is able to identify patients with $P M S 2$ mutations. Adding the location of CRC to the PREMM5 model improves the performance of the model for $P M S 2$ mutation carriers as well as its overall performance. These findings should be validated in large cohorts from population-based settings.

Acknowledgements We thank M Nielsen and JT Wijnen for their contribution in the data collection of this study.

\section{Compliance with ethical standards}

Conflict of interest None of the authors declare a conflict of interest.

Open Access This article is distributed under the terms of the Creative Commons Attribution 4.0 International License (http://creativecommons.org/licenses/by/4.0/), which permits unrestricted use, distribution, and reproduction in any medium, provided you give appropriate credit to the original author(s) and the source, provide a link to the Creative Commons license, and indicate if changes were made.

\section{References}

1. Lynch HT, de la Chapelle A (2003) Hereditary colorectal cancer. N Engl J Med 348(10):919-932

2. Watson P, Lynch HT (1993) Extracolonic cancer in hereditary nonpolyposis colorectal cancer. Cancer 71(3):677-685

3. de Jong AE, Hendriks YM, Kleibeuker JH et al (2006) Decrease in mortality in Lynch syndrome families because of surveillance. Gastroenterology 130(3):665-671

4. Jarvinen HJ, Aarnio M, Mustonen $\mathrm{H}$ et al (2000) Controlled 15 -year trial on screening for colorectal cancer in families with hereditary nonpolyposis colorectal cancer. Gastroenterology 118(5):829-834

5. Jarvinen HJ, Renkonen-Sinisalo L, Aktan-Collan K, Peltomaki P, Aaltonen LA, Mecklin JP (2009) Ten years after mutation testing for Lynch syndrome: cancer incidence and outcome in mutationpositive and mutation-negative family members. J Clin Oncol 27(28):4793-4797

6. Akiyama Y, Sato H, Yamada T et al (1997) Germ-line mutation of the hMSH6/GTBP gene in an atypical hereditary nonpolyposis colorectal cancer kindred. Cancer Res 57(18):3920-3923

7. Bronner CE, Baker SM, Morrison PT et al (1994) Mutation in the DNA mismatch repair gene homologue hMLH1 is associated with hereditary non-polyposis colon cancer. Nature 368(6468):258-261

8. Fishel R, Lescoe MK, Rao MR et al (1993) The human mutator gene homolog MSH2 and its association with hereditary nonpolyposis colon cancer. Cell 75(5):1027-1038

9. Nicolaides NC, Papadopoulos N, Liu B et al (1994) Mutations of two PMS homologues in hereditary nonpolyposis colon cancer. Nature 371(6492):75-80

10. Niessen RC, Hofstra RM, Westers H et al (2009) Germline hypermethylation of MLH1 and EPCAM deletions are a frequent cause of Lynch syndrome. Genes Chromosomes Cancer 48(8):737-744

11. Aaltonen LA, Salovaara R, Kristo P et al (1998) Incidence of hereditary nonpolyposis colorectal cancer and the feasibility of molecular screening for the disease. N Engl J Med 338(21):1481-1487

12. de la Chapelle A (2003) Microsatellite instability. N Engl J Med 349(3):209-210 
13. van Lier MG, Wagner A, van Leerdam ME et al (2010) A review on the molecular diagnostics of Lynch syndrome: a central role for the pathology laboratory. J Cell Mol Med 14(1-2):181-197

14. Cross DS, Rahm AK, Kauffman TL et al (2013) Underutilization of Lynch syndrome screening in a multisite study of patients with colorectal cancer. Genet Med 15(12):933-940

15. Julie $C$, Tresallet $C$, Brouquet $A$ et al (2008) Identification in daily practice of patients with Lynch syndrome (hereditary nonpolyposis colorectal cancer): revised Bethesda guidelines-based approach versus molecular screening. Am J Gastroenterol 103(11):28252835 (quiz 36)

16. Perez-Carbonell L, Ruiz-Ponte C, Guarinos C et al (2012) Comparison between universal molecular screening for Lynch syndrome and revised Bethesda guidelines in a large population-based cohort of patients with colorectal cancer. Gut 61(6):865-872

17. Van Lier MG, De Wilt JH, Wagemakers JJ et al (2009) Underutilization of microsatellite instability analysis in colorectal cancer patients at high risk for Lynch syndrome. Scand J Gastroenterol 44(5):600-604

18. Barnetson RA, Tenesa A, Farrington SM et al (2006) Identification and survival of carriers of mutations in DNA mismatch-repair genes in colon cancer. N Engl J Med 354(26):2751-2763

19. Chen S, Wang W, Lee S et al (2006) Prediction of germline mutations and cancer risk in the Lynch syndrome. JAMA 296(12):1479-1487

20. Kastrinos F, Uno H, Ukaegbu C et al (2017) Development and Validation of the PREMM5 Model for Comprehensive Risk Assessment of Lynch Syndrome. J Clin Oncol 35(19):2165-2172

21. Green RC, Parfrey PS, Woods MO, Younghusband HB (2009) Prediction of Lynch syndrome in consecutive patients with colorectal cancer. J Natl Cancer Inst 101(5):331-340

22. Khan O, Blanco A, Conrad P et al (2011) Performance of Lynch syndrome predictive models in a multi-center US referral population. Am J Gastroenterol 106(10):1822-1827 (quiz 8)

23. Monzon JG, Cremin C, Armstrong L et al (2010) Validation of predictive models for germline mutations in DNA mismatch repair genes in colorectal cancer. Int J Cancer 126(4):930-939

24. Pouchet CJ, Wong N, Chong G et al (2009) A comparison of models used to predict MLH1, MSH2 and MSH6 mutation carriers. Ann Oncol 20(4):681-688

25. Ramsoekh D, van Leerdam ME, Wagner A, Kuipers EJ, Steyerberg EW (2009) Mutation prediction models in Lynch syndrome: evaluation in a clinical genetic setting. J Med Genet 46(11):745-751

26. Tresallet C, Brouquet A, Julie C et al (2012) Evaluation of predictive models in daily practice for the identification of patients with Lynch syndrome. Int J Cancer 130(6):1367-1377
27. Win AK, Macinnis RJ, Dowty JG, Jenkins MA (2013) Criteria and prediction models for mismatch repair gene mutations: a review. J Med Genet 50(12):785-793

28. Boland CR, Thibodeau SN, Hamilton SR et al (1998) A National Cancer Institute Workshop on Microsatellite Instability for cancer detection and familial predisposition: development of international criteria for the determination of microsatellite instability in colorectal cancer. Cancer Res 58(22):5248-5257

29. Suraweera N, Duval A, Reperant M et al (2002) Evaluation of tumor microsatellite instability using five quasimonomorphic mononucleotide repeats and pentaplex PCR. Gastroenterology 123(6):1804-1811

30. van der Klift HM, Tops CM, Bik EC et al (2010) Quantification of sequence exchange events between PMS2 and PMS2CL provides a basis for improved mutation scanning of Lynch syndrome patients. Hum Mutat 31(5):578-587

31. Van Hoorde K, Vergouwe Y, Timmerman D, Van Huffel S, Steyerberg EW, Van Calster B (2014) Assessing calibration of multinomial risk prediction models. Stat Med 33(15):2585-2596

32. Lee SY, Kim DW, Shin YK et al. (2015) Validation of prediction models for mismatch repair gene mutations in Koreans. Cancer Res Treat. doi:10.4143/crt.2014.288

33. Kastrinos F, Ojha RP, Leenen C et al. (2016) Comparison of prediction models for Lynch syndrome among individuals with colorectal cancer. J Natl Cancer Inst. doi:10.1093/jnci/djv308

34. Giardiello FM, Allen JI, Axilbund JE et al (2014) Guidelines on genetic evaluation and management of Lynch syndrome: a consensus statement by the US Multi-Society Task Force on Colorectal Cancer. Gastroenterology 147(2):502-526

35. Vasen HF, Blanco I, Aktan-Collan K et al (2013) Revised guidelines for the clinical management of Lynch syndrome (HNPCC): recommendations by a group of European experts. Gut 62(6):812-823

36. Barzi A, Sadeghi S, Kattan MW, Meropol NJ (2015) Comparative effectiveness of screening strategies for Lynch syndrome. J Natl Cancer Inst. doi:10.1093/jnci/djv005

37. Ladabaum U, Wang G, Terdiman J et al (2011) Strategies to identify the Lynch syndrome among patients with colorectal cancer: a cost-effectiveness analysis. Ann Intern Med 155(2):69-79

38. Palomaki GE, McClain MR, Melillo S, Hampel HL, Thibodeau SN (2009) EGAPP supplementary evidence review: DNA testing strategies aimed at reducing morbidity and mortality from Lynch syndrome. Genet Med 11(1):42-65

39. Vergouwe Y, Steyerberg EW, Eijkemans MJ, Habbema JD (2005) Substantial effective sample sizes were required for external validation studies of predictive logistic regression models. J Clin Epidemiol 58(5):475-483 